\title{
Alarm Management Technology and Its Progress in Process Industries
}

\author{
Jinqiu $\mathrm{Hu}^{*}$, Shuang Cai and Laibin Zhang \\ China University of Petroleum (Beijing), Beijing, China \\ ${ }^{*}$ Corresponding author
}

\begin{abstract}
With continuous progress of science and technology, the alarm configurations in process industries have become easier and huger. Due to the complexity of the process system and the unreasonable design of the alarm system, large and invalid alarms have been produced. Operators can be overwhelmed by the large amount of alarms and not take effective measures to abnormal conditions, leading to the occurrence of accidents. In order to effectively manage the alarm system and ensure the safe operation in industrial process, many scholars have made a lot of researches on the related issues of alarm management in process industries. By evaluating the existing methods, the problems in this field and the directions that deserve further research are pointed out in this paper. Finally, prospects are made with respect to the future development of alarm system management.
\end{abstract}

Keywords-alarm management; rational design; multivariate alarm analysis; causal identification

\section{INTRODUCTION}

Excessive or even ineffective alarms occur owing to the complexity of the process system and the unreasonable design of the alarm system. A large number of alarm information cannot make operators take effective measures to the abnormal situations in time and then result in process variables deviating from their design ranges and potential undesired outcomes, even serious consequence. Therefore, alarm systems play significant roles in maintaining plant safety and keeping the process within normal operating ranges.

Currently, alarm floods are the most common and the most difficult problem of the alarm system. Additionally, the false alarm and missed alarm probabilities are the most important indicators to assess the performance for alarm system in process industries. Many scholars have made a lot of research on the related issues of alarm management in process industries. This paper reviewed several research hotspots on these issues with the following contributions: (i) Rational design of alarm system, including delay-timer and deadband design, alarm threshold optimization, and alarm priority ranking. Process time series are analyzed by these methods to generate a more effective alarm signal so that the accuracy and rapidity of alarms can be improved; (ii) Multivariate alarm analysis and causal identification, including alarm correlation, root-cause analysis and alarm floods pattern matching. These methods are proposed to deal with multi variable alarm, in particular, restrain the alarm flooding and improve the simplicity and practicality of alarms. By evaluating the existing methods, the problems in this field and the directions that deserve further research are pointed out. Finally, prospects are made with respect to the future development of alarm system management.

\section{RELATED ISSUES OF ALARM MANAGEMENT IN PROCESS INDUSTRIES}

Typically, Alarm flooding is a pressing problem of alarm system management. Most of the alarms during alarm flooding are nuisance. Chattering alarms, fleeting alarms and stale alarms are all common forms of nuisance alarms. Different types of alarms (1) are defined as shown in Table 1.

TABLE I. DEFINITION OF DIFFERENT TYPES OF ALARMS

\begin{tabular}{|c|c|}
\hline Alarm type & Definition \\
\hline Nuisance alarm & $\begin{array}{l}\text { An alarm that annunciates excessively, unnecessarily, } \\
\text { or does not return to normal after the correct response } \\
\text { is taken }\end{array}$ \\
\hline Chattering alarm & $\begin{array}{l}\text { An alarm that repeatedly transitions between the alarm } \\
\text { state and the normal state in a short period of time. }\end{array}$ \\
\hline Fleeting alarm & $\begin{array}{l}\text { Fleeting alarms are similar short-duration alarms that } \\
\text { do not immediately repeat. }\end{array}$ \\
\hline Stale alarm & $\begin{array}{l}\text { An alarm that remains in the alarm state for an } \\
\text { extended period of time. }\end{array}$ \\
\hline False alarm & $\begin{array}{c}\text { A false alarm is an alarm that is raised when the } \\
\text { variable is behaving normally }\end{array}$ \\
\hline Missed alarm & $\begin{array}{l}\text { a missed alarm occurs when the process is behaving } \\
\text { abnormally but no alarm is raised }\end{array}$ \\
\hline
\end{tabular}

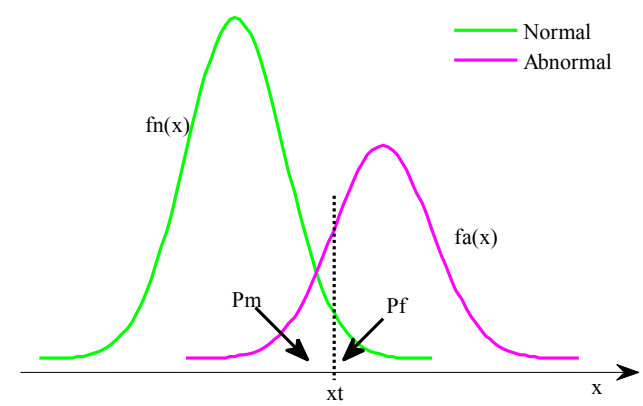

FIGURE I. PROBABILITY DENSITY FUNCTION OF A PROCESS VARIABLE

Further it is known that the alarm systems of process industries generate many false alarms and missed alarms (corresponding definitions shown in table 1). Unreasonably assigned thresholds may result in frequent false alarms and missed alarms. Assume that the probability density functions of the process variable $x$ are shown in Fig. 1 , where $f_{n}(x)$ and $f_{a}(x)$ are its functions in normal and abnormal situations, $x_{t}$ is the 
high alarm threshold. Since part of the distribution of normal data that is the left side of the curve falls above the trip point, some false alarms will be raised. According to the minimum error probability and Bayesian decision theory, the false alarm probability ( $\mathrm{p}_{\mathrm{f}}$ in Fig.1) is the area under the distribution curve of normal data and higher than the trip point, as shown in Eq. (1). A number of alarms will also be missed with this trip point, for all the abnormal data points do not fall above the trip point. The missed alarm probability ( $\mathrm{p}_{\mathrm{m}}$ in Fig. 1 ) is the area under the distribution curve of abnormal data and lower than the trip point, as shown in Eq. (2). It can be seen that the false alarm probability decreases but the missed alarm probability increases by increasing the trip point (2).

$$
\begin{aligned}
& p_{f}=\int_{x_{t}}^{+\infty} f_{n}(x) d x . \\
& p_{m}=\int_{-\infty}^{x_{t}} f_{a}(x) d x .
\end{aligned}
$$

Nowadays, the rational design of alarm system is critical in determining whether the process industry works properly or it distracts the operators with raising too many nuisance and false alarms. Moreover, alarm floods have always been serious hazards in process industries since they overwhelm operators with large amount of alarm messages raised within a short period of time. Researches on multivariate alarm analysis and causal identification have become more and more significant for solving alarm floods problems. Related research methods about these problems are investigated and summarized in the following section.

\section{RATIONAL DESIGN OF ALARM SYSTEM}

A good alarm should have many characteristics, such as accuracy, timeliness, clear priorities, understandability, etc. In order to optimize the alarm threshold and avoid the occurrence of alarm floods, some scholars take the false alarm $\left(\mathrm{p}_{\mathrm{f}}\right)$ and missed alarm probabilities $\left(\mathrm{p}_{\mathrm{m}}\right)$ as the design indices. Receiver operating characteristic (ROC) is applied to analyzing the contradictory relationship between them and accomplish the optimal design of alarm system. The relationship between $p_{f}$ and $\mathrm{p}_{\mathrm{m}}$ can be shown as a ROC curve (shown in Fig. 2). The dotted line represents the tangent of the curve. The best limit is chosen according to an optimization, as shown in Eq. (3):

$$
\underset{x_{0}}{\operatorname{Min}}\left(\alpha p_{m}+(1-\alpha) p_{f}\right)
$$

where $\alpha$ is a weighting factor subjectively chosen to reflect the degree of importance. The choice of the value of $\alpha$ entails a difficult value judgment of the relative importance of false alarm and missed detection. One has to find out an acceptable trade-off between $p_{m}$ and $p_{f}$, which is typically the tangential point $\mathrm{x}_{0}$ of ROC curve and one of the lines with slope $\alpha /(1-$ $\alpha)$. Filter, delay-timer and deadband are applied to analyzing the effects of different design parameters on the accuracy.
Moreover, alarm threshold optimization and alarm priority ranking also play an important role in rational design of alarm system.

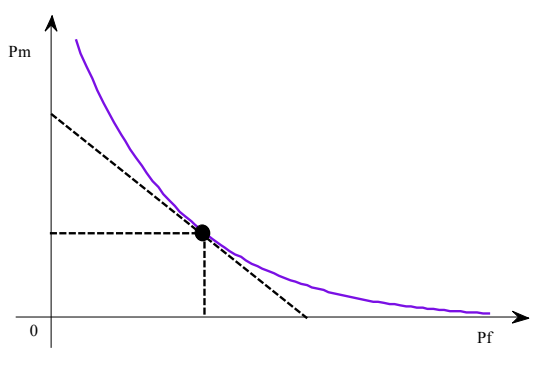

FIGURE II. ROC CURVE

\section{A. Delay-timer and Deadband}

To reduce false and nuisance alarms, techniques like filters, deadbands, and delay-timers are widely used in process industry. Among all these methods, delay-timers are commonly used for its simplicity and efficiency. In (3), a design method is proposed to balance false alarm rate, missed alarm rate and expected detection delays. In (4), the relation between optimal alarm threshold and deadband is discussed. Two equations are proposed to estimate the optimal threshold with respect to the deadband and history of the process variable. However, using these techniques introduces some delay in raising the alarm (detection delay).

\section{B. Alarm Threshold Optimization}

In current process industries, alarm threshold optimization is a significant part of alarm system rationalization. In (5), the change detection algorithm is proposed to get the adaptive alarm thresholds, it kept the false alarm rate constant, but failed to consider the influence of missed alarms. Nevertheless, existing techniques for the design of alarm systems mostly focus on one steady state of operation and cannot effectively deal with alarm floods during transitions. In (6), Bayesian estimation based on dynamic alarm management (BEDAM) method is applied to determine the dynamic alarm thresholds during transitions. In (2), a new method is proposed for the multivariate alarm thresholds optimization based on FAP, MAP and correlation analysis. Since the proposed method just sets one limit for every alarm variable, how to get both the optimal high and low alarm thresholds is a question that is worth discussing in the future.

\section{Alarm Priority Ranking}

Alarm priority indicates the importance of the alarm. The method for priority assignment is applied to the rationalized alarm. Effective prioritization typically results in higher priorities chosen less frequently than lower priorities (1). Effective use of alarm priority can enhance the ability of the operator to manage alarms and provide proper response.

In (7), an integrated model consisting of the probability $(P)$, the impact $(I)$ of the potential hazards, and the process safety time $(\mathrm{t})$ is presented to prioritize the raised alarms. Considering the process safe time for the alarm to be $1 \mathrm{~min}$, the risk value (R) of the alarm can be obtained by Eq. (4). The proposed 
approach incorporates the process safety time rather than the operator's response time, which practically represents the real cases at the time when the alerts are raised. How to design the above three factors according to different applied objects is a question which is worth studying. An approach in (8) is to create revised Likert scale based on the concept of relative importance index, from which the alarm priorities are determined. In (9), the Alarm Prioritization System (ALAP) is developed to calculate the severity of each alarm, categorize the alarms and rank the alarms based on its priority. The system will get the trend data from DCS and analyze it. The rules in the knowledge base will be combined to carry out fuzzy reasoning and achieve priority classification. Furthermore, the capabilities of self-learning and self-tuning can be discussed so that the prototype system can perform automatic diagnostics of the alarms patterns in the near future.

$$
R=P \times I \times 100^{(1-t / 60)}
$$

\section{Multivariate Alarm Analysis And CAUSAL IDENTIFICATION}

With the wide use of DCS, PLC and FCS, many alarm tags are now very easy to be configured on every possible process variables and state variables with almost no extra costs and changes. Moreover, most processes are multivariate and relate to material flow, energy flow, signal flow and information flow (8). Abnormalities can propagate from one part to other parts of the plants via connecting paths. The correlation and causality can also produce many alarms. As a result, plant operators frequently experience a large number of redundant and nuisance alarms. In this circumstance, operators' focuses may be distracted from the important alarms and alarm root causes, which are most likely to be submerged in alarm floods. All these issues necessitate a good strategy for multivariate alarm analysis and causal identification including correlation alarm, root-cause analysis and alarm floods pattern matching.

\section{A. Alarm Correlation Analysis}

In order to identify the causality and dependency among variables, correlation is an efficient and important criterion. Correlation coefficient is often used to describe the relationships between two time series of process variables.

The correlation of process data and alarm data is analyzed in (2) and used to estimate the optimal thresholds. In (8), partial correlation coefficient analysis is used to quantify the direct correlation between any two variables and establish an interpretive structural model for abnormality propagation path identification and root-cause analysis. In (10), the correlation delay between alarm signals is estimated so that it can be separated from the occurrence delays, which is used to detect correlated alarms, to obtain real occurrence delays (ROD). Thereafter, a statistical test based on the ROD is proposed to determine whether two alarm signals are correlated or not, and the Pearson's correlation coefficient is applied to quantifying the correlation level.

\section{B. Causal Identification and Alarm Root-cause Analysis}

Causal alarm is a main reason for multiple alarms and even alarm floods. For causal alarms, the root-cause alarm need to be analyzed so that the problem of alarm floods can be solved from the source. On the one hand, the topological model of process can be established based on process knowledge to determine the process connection path and fault propagation path. It is helpful to causal alarm identification and root-cause analysis, such as signed directed graph (SDG) (11, cause-effect model (CE) (12, multilevel flow model (MFM), etc. On the other hand, the data driven method can be used to realize the identification of cause and effect alarm, such as information theory method (transfer entropy, direct transfer entropy) (13), time delay correlation analysis, Bayesian network modeling (14), etc. So that the causal alarm sequence can be identified to find the alarm source and fundamentally suppress nuisance alarms and alarm floods.

Knowledge modeling method is more practical, but the model is easy to be affected by the subjective judgment. Data driven analysis method avoids the trifles of process knowledge modeling considering all variables, but the delay correlation cannot analyze the nonlinear correlation, which can only approximately show the causal relationship, and does not necessarily represent the real cause and effect relationship. Transfer entropy method is applicable to both linear and nonlinear relationship. It can find the loop connection paths between variables, direct transfer entropy may also determine whether there is a direct transfer path, but it cannot detect the size of time delay and heavily depend on the probability density function, and the computational complexity is large. Bayesian network can reflect the randomness and uncertainty of real process, but it need to use the data of all modes. These data are sometimes difficult to get, and the physical interpretation of the probability is not direct.

\section{Alarm Floods Pattern Matching}

Many pattern mining algorithms have been applied to facilitating the research of consequence alarms in alarm floods. Historical alarm sequences can be applied to extracting time patterns of alarms. It can be examined by sequential pattern matching. This idea is used in (16) to cluster frequent occurring subsequences in alarm logs and identify alarms with causal relations. Then the alarm systems can be redesigned for eliminating alarm floods. Similar historical alarm floods can be utilized to draw out representative information. In (17), a pattern growth approach is used to obtain patterns in alarm floods. However, the proposed approach is sensitive to disturbances so that the patterns in sequences have to be exactly the same in order to be recognized. A modified SmithWaterman algorithm is proposed in (15) for local alignment of two alarm flood sequences so that their similarity scores can be obtained and common alarm sequence segments could be extracted. The novel method tolerates to disturbances and order changes as well but is limited to pairwise usage. In (18), the proposed algorithm is an extension of (15) to the multiple sequence case. Significant innovations in similarity scoring, dynamic programming, back tracking procedure, and alignment generation are made to achieve the expansion. 


\section{CONCLUSION AND PROSPECTS}

This paper reviewed several research approaches on related issues of alarm management in process industries. By evaluating the existing research results of the alarm system and the advantages and disadvantages, some of the problems that deserve further research and the development trend of the alarm management are revealed.

1) Currently, the alarm threshold design and optimization mostly consider the false alarm rate, missed alarm rate and detection delay as the objective function, with little regard for the time delay and correlation among variables. The correlation among alarm variables will be changed with the different alarm threshold. Moreover, time delay information is helpful to the reasonable design of alarm threshold, which can reduce false alarms and nuisance alarms. Therefore, on the basis of the original objective function, considering the time delay and related information, the multi objective function can be established to determine the optimal threshold value. Additionally, when abnormal conditions or raw materials change, the alarm threshold should be adjusted dynamically to realize the online adjustment of the threshold.

2) Research on the alarm prioritization is very few, but its importance cannot be ignored. The current methods in industrial practice are mostly qualitative, based on the understanding of severity in potential risk and a crude approximation of safety time in handling alarms. Therefore, quantitative approaches should be combined with the qualitative approaches in the future. The importance of the alarm may change with state transations. So the online adjustment of priorities need to be considered in the future.

3) Knowledge modeling method and data driven analysis method are two common methods for multivariate alarm analysis. Knowledge modeling method is more practical, but the model is easy to be affected by the subjective judgement. Data driven analysis method avoids the trifles of process knowledge modeling considering all variables, but the delay correlation, the nonlinear correlation, randomness and uncertainty cannot be considered synthetically in these methods. Hence, it is necessary to utilized qualitative process knowledge and quantitative data simultaneously in analyzing correlations of multi variables. And the nonlinearity-based methods need to be exploited in complex systems.

4) Many pattern mining algorithms have been applied to facilitating the research of sequence alarms in alarm floods. However, the most algorithms are offline with the slow computation speed, especially for long sequences in alarm floods. Therefore, the algorithm with faster computing power should be applied to matching an online alarm sequence to its similar ones in the historical database.

5) A predictive alarm to indicate incoming abnormalities can provide operators with more time to take proactive actions. If an online sequence is identified as similar to a pattern, the prediction of upcoming alarms will be forecasted based on the pattern in the database. The alarm messages contained in the pattern but not included in the online sequence can be given as predictions. More early warning methods can be considered in alarm management.

\section{ACKNOWLEDGMENT}

This project is supported by Natural Science Foundation of China (Grant No. 51574263); Science Foundation of China University of Petroleum,Beijing (Grant No.2462015YQ0403); China University of Petroleum(Beijing) Youth Innovation Team C program (Grant No.C201602)

\section{REFERENCES}

[1] ISA, ANSI/ISA-18.2: Management of Alarm Systems for the Process Industries. International Society of Automation. Durham, NC, USA, 2009.

[2] L Han, H Gao, Y Xu, et al. Combining FAP, MAP and correlation analysis for multivariate alarm thresholds optimization in industrial process. Journal of Loss Prevention in the Process Industries, 2016, vol. 40:, pp. 471-478.

[3] N A Adnan, Y Cheng, I Izadi, et al. Study of generalized delay-timers in alarm configuration. Journal of Process Control, 2013, vol. 23, pp. 382395.

[4] E Naghoosi, I Izadi, T Chen. A study on the relation between alarm deadbands and optimal alarm limits. 2011 American Control Conference (ACC 2011), IEEE. 2011, pp. 3627-3632.

[5] G Verdier, N Hilgert, J P Vila. Adaptive threshold computation for CUSUM-type procedures in change detection and isolation problems. Computational Statistics \& Data Analysis, 2008, vol. 52, pp. 4161-4174.

[6] J Zhu, Y Shu, J Zhao, et al. A dynamic alarm management strategy for chemical process transitions. Journal of Loss Prevention in the Process industries, 2014, vol. 30, pp. 207-218.

[7] Y Chang, F Khan, S Ahmed. A risk-based approach to design warning system for processing facilities. Process Safety and Environmental Protection, 2011, vol. 89, pp. 310-316.

[8] $\mathrm{H} \mathrm{Gao}, \mathrm{Y} \mathrm{Xu}, \mathrm{X} \mathrm{Gu}$, et al. Systematic rationalization approach for multivariate correlated alarms based on interpretive structural modeling and Likert scale. Chinese Journal of Chemical Engineering, 2015, vol. 23, pp. 1987-1996.

[9] O Foong, S Sulaiman, D Rambli, et al. ALAP: Alarm prioritization system for oil refinery. Proc. of the World Congress on Engineering and Computer Science. 2009.

[10] W Hu, J Wang, T Chen. A new method to detect and quantify correlated alarms with occurrence delays. Computers \& Chemical Engineering, 2015, vol. 80, pp. 189-198.

[11] F Yang, L S Sirish, D Xiao. Signed directed graph modeling of industrial processes and their validation by data-based methods. Control and Fault-Tolerant Systems (SysTol), 2010 Conference on. IEEE, 2010, pp. 387-392.

[12] Y Wan, F Yang, N Lv, et al. Statistical root cause analysis of novel faults based on digraph models. Chemical Engineering Research and Design, 2013, vol. 91, pp. 87-99.

[13] M Bauer, J W Cox, M H Caveness, et al. Finding the direction of disturbance propagation in a chemical process using transfer entropy. Control Systems Technology, IEEE Trans, 2007, vol. 15, pp. 12-21.

[14] L Abele, M Anic, T Gutmann, et al. Combining Knowledge Modeling and Machine Learning for Alarm Root Cause Analysis. MIM. 2013:, pp. 1843-1848.

[15] Y Cheng, I Izadi, T Chen. Pattern matching of alarm flood sequences by a modified Smith-Waterman algorithm. Chemical Engineering Research and Design, 2013, vol. 91, pp. 1085-1094.

[16] S Lai, T Chen. Methodology and Application of Pattern Mining in Multiple Alarm Flood Sequences. IFAC-PapersOnLine, 2015, vol. 48, pp. 657-662.

[17] J Folmer, B Vogel-Heuser. Computing dependent industrial alarms for alarm flood reduction. Systems, Signals and Devices (SSD), 2012 9th International Multi-Conference. IEEE, 2012, pp. 1-6.

[18] S Lai, T Chen. A method for pattern mining in multiple alarm flood sequences. Chemical Engineering Research and Design, 2015. 